\title{
The Study of Gait Cycle Stability Using a Five-Link Inverted Pendulum Model: First Developments
}

\author{
Luís Moreira ${ }^{1}$, Daniel Sanz-Merodio ${ }^{2}$, Joana Figueiredo ${ }^{1}$, Cristina P. Santos ${ }^{1}$, and Elena Garcia ${ }^{2}$
}

\begin{abstract}
Gait cycle has been the target of many studies in the last decades in order to identify the normal walking patterns of humans. The usefulness of these studies is of utmost importance to construct mathematical models that are capable to achieve stability during walking. Based on this fact, it seems justified the present effort to construct a model to simulate a complete stride in healthy humans. Thus, in this work, a five-link inverted pendulum model in the sagittal plane with 5 degrees of freedom (DOFs) based on Euler-Lagrange equations is being constructed with the main objective to concern stability during gait cycle. To do that, this study was divided into two parts: the first one is represented by this article and it is regarding to the model construction and data collection; the second part is relative to a future work and it concerns to the model validation and an analysis of the stiffness variation with the power and speed during gait cycle. Regarding to the results of the present study, good correlations were obtained between the data collected and the data presented in literature, which demonstrates a good data acquisition.
\end{abstract}

\section{INTRODUCTION}

The gait cycle is characterized for continuous repetitions of successive strides and it is divided into two phases: stance and swing. During walking, the first term corresponds to the period in which the foot is on the ground (until 60\% of gait cycle), while the second corresponds to the period in which the foot is in the air (the remaining 40\%) [1]. In the last years, gait analysis has become the focus of many studies in order to identify which are the normal human gait patterns. Some musculature or neurological diseases imply that some people cannot walk without some support, such as exoskeletons or orthoses [2]. For that, a correct simulation of the motion of the lower limbs is useful to analyze the angles, forces and moments which each joint should perform during walking motion in order to ensure stability. Thus, in this study, a fivelink inverted pendulum model, in the sagittal plane, based on Euler-Lagrange equations is proposed in order to analyze the kinematic and the dynamic lower limbs motion during a complete stride. It was followed the inverted pendulum approach due to energy reasons: according with [3], there is energy conservation if the behavior of the stance leg is identical to the behavior of an inverted pendulum; the same happens if the behavior of the swing leg is identical to the behavior of a pendulum. Between Euler-Lagrange and Newton-Euler mechanics, it was chosen the first one because the prior knowledge of the ground reaction force (GRF) is not necessary, that is, Euler-Lagrange approach only requires energy calculations [4].

\footnotetext{
${ }^{1}$ Luís Moreira, Joana Figueiredo, and Cristina P. Santos are with Center of MicroElectroMechanical Systems (CMEMS), University of Minho, Portugal, \{a74999@alunos, id6003@alunos, cristina@dei\}.uminho.pt
}

Regarding to the kinetic and kinematic data to introduce in the model, the most common way to get them is using motioncapture systems and reflective markers. However, should be noted that the placement of these markers is very delicate in order to obtain good data. To guarantee feasibility regarding to the data acquired, it is possible to calculate the value of the correlation existent between the data collected and the literature. In this sense, the Spearman's correlation coefficient is a powerful tool which evaluates the strength of a linear monotonic relationship between data. This coefficient varies between -1 and 1 , where values near zero indicate a very weak correlation and values above 0.8 indicate a very strong correlation. Values below zero indicate different monotonies, that is, if one variable in study increases, the other decreases and vice-versa. The Pearson's correlation can also be used, but it is necessary to pay attention to the distribution of the data, i.e., if the data are distributed with a null mean and a standard deviation of one [5].

Thus, regarding to the entire work, this one will be divided into two parts: the first one integrates all the data collection and model construction (discussed in this article); the second one is relative to the model validation with the data collected and it is also relative to a study of the stiffness variation with the power and speed during walking (as future work). In this sense, in section II will be presented an explanation of the mathematical model used and in section III will be presented all the procedure to collect data with the motion-capture system (MCS). Section IV will present the results and discussion of this work, and finally, section V concerns to the conclusions of the present work, as well as some future perspectives.

\section{MATHEMATICAL ANALYSIS}

\section{A. Lower Limbs Definitions}

The five-link model developed in this work is composed by two rigid legs connected to the torso through the hip joint. Each leg is constituted by two links, shank and thigh, connected to the knee joint. As first step to obtain the equations of motion for the five-link inverted pendulum model in the sagittal plane, it was necessary to consider each part of the human body as links or rigid bodies: shank link, thigh link and HAT (head, arms and trunk) link. Thus, it is necessary to take in account three joints, being ankle, knee and hip joint. So, considering both legs, there are five joints (two ankles, two knees and one hip joint) with five DOFs, one DOF/joint. With respect to the origin of the model, this one was placed in the ankle joint of the foot that was in contact with the floor. Thus, considering just one leg in the sagittal plane, there are three

\footnotetext{
${ }^{2}$ Daniel Sanz-Merodio and Elena Garcia are with Marsi Bionics S.L., Spain, \{daniel.sanz, elena.garcia\}@marsibionics.com
} 
angles: $\theta_{1}, \theta_{2}$ and $\theta_{3}$, designed by ankle angle, knee angle and hip angle, respectively. The first angle corresponds to the angle between the shank segment and the vertical world axis. The second one is the angle between the thigh segment and the prolongation of the shank and the last one concerns to the angle between the torso segment and the prolongation of the thigh. In Fig. 1 is possible to see the five rigid bodies, the five angles and the world axis that was placed in the ankle joint.

\section{B. Derivation of the Equations of Motion}

The equations of motion for the five-link inverted pendulum can be written as (1). [6]

$$
\mathrm{M}(\theta) \ddot{\theta}+\mathrm{C}(\theta, \dot{\theta})+\mathrm{G}(\theta)=\tau
$$

Where $M \in \mathfrak{R}^{\mathrm{n} \times \mathrm{n}}$ is Mass matrix, $\mathrm{C} \in \mathfrak{R}^{\mathrm{n} \times \mathrm{n}}$ is Coriolis matrix, $G \in \mathfrak{R}^{\mathrm{n} \times 1}$ represent the Gravitational forces, $\dot{\theta}$ represents the vector of angular joint velocities $\left(\dot{\theta}=\left[\dot{\theta}_{1}, \dot{\theta}_{2}\right.\right.$, $\left.\left.\dot{\theta}_{3}\right]\right), \ddot{\theta}$ represents the vector of joint accelerations $\left(\ddot{\theta}=\left[\ddot{\theta}_{1}, \ddot{\theta}_{2}\right.\right.$, $\left.\ddot{\theta}_{3}\right]$ ) and $\tau \in \mathfrak{R}^{\mathrm{n} \times 1}$ represents the torque. Since there are $3 \mathrm{DOF}$ (degrees of freedom) in the sagittal plane, $\mathrm{n}$ is equal to 3 . Thus, for each link, one equation is obtained, i.e., three equations in the sagittal plane. To get these three equations is required to follow a procedure. The first step is to obtain the cartesian positions for each joint and for each center of mass of each link, considering the angles regarding to the origin of the system and the lengths of each segment. These lengths can be measured or can be consulted in [7]. Then, it is necessary to get the kinetic and potential energy for each segment in order to obtain the Lagrangian (2). For that, it is required to derivate the cartesian positions and the joint angles regarding to the time, obtaining the linear and angular velocity, respectively.

$$
\mathrm{L}_{\mathrm{i}}=\mathrm{K}_{\mathrm{i}}-\mathrm{P}_{\mathrm{i}}
$$

With $\mathrm{i}=1,2,3$. Once the Lagrangian has been determined, the next step is to obtain the torque for each link, given by (3).

$$
\tau_{\mathrm{i}}=\frac{\mathrm{d}}{\mathrm{dt}}\left(\frac{\partial \mathrm{L}}{\partial \dot{\theta}_{\mathrm{i}}}\right)-\frac{\partial \mathrm{L}}{\partial \theta_{i}}-\sum_{\mathrm{j}}\left(\lambda_{\mathrm{j}} \frac{\partial \mathrm{f}_{\mathrm{j}}}{\partial \theta_{\mathrm{i}}}\right)
$$

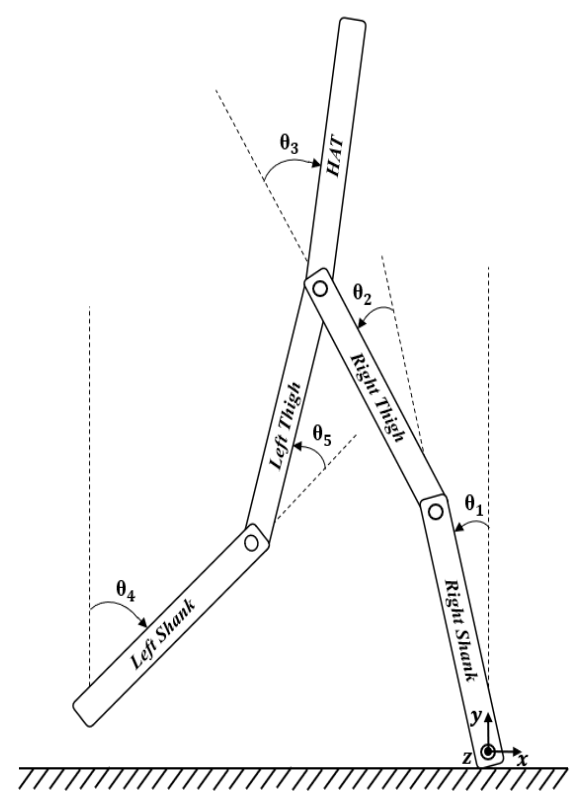

Figure 1. Rigid bodies and angles of the five-link inverted pendulum model.
With $\mathrm{i}=1,2,3$ and $\mathrm{j}$ is the number constraints. Note that (3) is only used in double support phases (when both feet are in the ground). For single support phases, (3) is used without the third term of the right side. Then, developing the equation in order to get the Mass, Coriolis and Gravitational matrix, it is possible to obtain (1) $[\mathbf{4 , 6}]$. At this point, it is possible to introduce the joint angles, velocities and accelerations and analyse the torques provided by the model. As previous mentioned, the validation of the model will be done in a future work.

\section{PRocedures to Data Collection}

In order to validate the mathematical model, a database of kinematic and kinetic data for lower body was collected during gait cycle from healthy volunteers. It is important to note that ethical approval for this study was given by Marsi Bionics S.L. and CSIC-UPM, based on Helsinki Declaration.

\section{A. Volunteers}

The study was performed with 11 adult volunteers of both genders (age $31 \pm 7.93$ years, height $171.4 \pm 9.21 \mathrm{~cm}$ and mass $70,8 \pm 13.6 \mathrm{~kg}$ ), with no clinical history or evidence of any type of physical and psychological disorders that could interfere with their gait patterns. To guarantee this phenomenon, each volunteer completed a medical questionnaire before the experiments. To all participants was asked to wear fitting clothes in order to reduce the movement of the reflective markers. It should be noted that the volunteers must not have shoes to perform the experiments.

\section{B. Materials and Data Acquisition}

Regarding to the human motion, a lower-body marker set was adopted, integrating sixteen reflective markers (with 14$\mathrm{mm}$ of diameter) placed on pelvis and lower limbs and were used eight cameras (Vero; Vicon - Motion Capture Systems, Oxford, UK) to obtain three-dimensional data. Each camera can give $330 \mathrm{fps}$ (frames per second) and it is able to capture movements with very low latency. The cameras were distributed around the laboratory to correctly capture the action volume. In the centre of the capture volume were placed two force plates $(0.5000 \times 0.2985-\mathrm{m}$ 9260AA3 model; Kistler, Winterthur, Switzerland) embedded in the floor in order to obtain the kinetic data. The configuration of the force plates on the floor was studied. Initially, both platforms were placed next to each other. However, in this case, two things happened: first, some volunteers changed their pattern of walking, unconsciously, in order to put each foot in each force plate; second, some volunteers placed the second step out of the second force plate, indicating that the force plates had to be closer to each other. Thus, it was decided to place the force plates crossed (as shown in Fig. 2), verifying that there was no change in the gait pattern. With respect to the kinematic data, these were acquired at $100 \mathrm{~Hz}$, while the ground reaction forces from both force plates were acquired at $200 \mathrm{~Hz}$. Prior to data collection, the synchronization of the system was done.

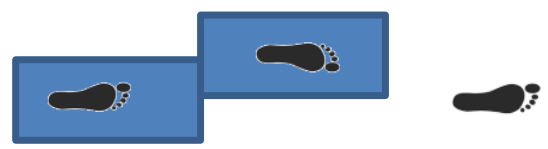

Figure 2. Ideal configuration found for a correct measurement. 


\section{System Calibration}

Before collecting data, it was necessary define a rectangular action perimeter around the force plates with reflective markers $(1.80 \times 0.850-\mathrm{m})$, being these the ideal dimensions to perform a complete stride, according with [8]. Then, the focus, light and zoom were adjusted to the defined perimeter to see all the markers in the right way. It is important to note that it was necessary to take in account the maximum leg length of the population in order to adjust the parameters (focus, light and zoom) of each camera to see the markers in the pelvis. Once these parameters were set, all the markers were removed, as well as the reflections of the environment. As last two steps, all cameras were calibrated with a wand and it was defined the origin of the system. These procedures of calibration were done based on [9]

\section{Subject Calibration}

Regarding to the marker-set adopted, it was used reflective markers placed in specific positions, according to [10]. It is important to highlight that to distinguish both legs, the RTHI (right thigh) and the LTHI (left thigh) markers must have different heights in the coronal plane, as well as RTIB (right tibia) and LTIB (left tibia) markers. This phenomenon can be visualized in Fig. 3. After the marker placement, it was measured the body height and the body mass, as well as leg and shank length and the knee and ankle width. Age and gender were also noted. After anthropometric data have been registered and introduced in the system, it was asked to each volunteer to stay in a comfortable position, with both hands crossed in front of the chest and looking ahead for three seconds. This procedure was done to perform a static anatomical-calibration in order to adjust all the recordings to the dimensions of each volunteer. Thus, the conditions to collect data were congregated.

\section{E. Data Collection}

Once the placement of the markers and the calibration of the system and the subjects have been performed, it was asked to the participants to walk in a rectilinear trajectory with a comfortable speed, looking in front. At the beginning of the first data collections, there were some gaps in the markers of both thighs hidden by both hands. In this sense, it was asked to each volunteer to walk with both hands in front of the chest. Each volunteer performed four trials separately.

\section{F. Data Processing}

To the data acquired with the motion-capture system, it was applied some procedures of processing. First, the kinematic data was filtered with a zero-phase fourth-order low pass Butterworth. In [11] was studied the relation between frequency and velocity of people walking and it was concluded that the people walk with an average frequency of 2-Hz. However, using the mentioned filter with a cut frequency of $2-\mathrm{Hz}$, it was noticed that the output signal was attenuated. According to [12], 99\% of the signal is below of 7-Hz. Thus, a filter with a cut frequency of 6-Hz was applied. This zero-phase digital filter was used because it doesn't introduce phase in the filtered signal.

Regarding to the force plates data, these ones were filtered with a zero-phase fourth-order low pass Butterworth with cut

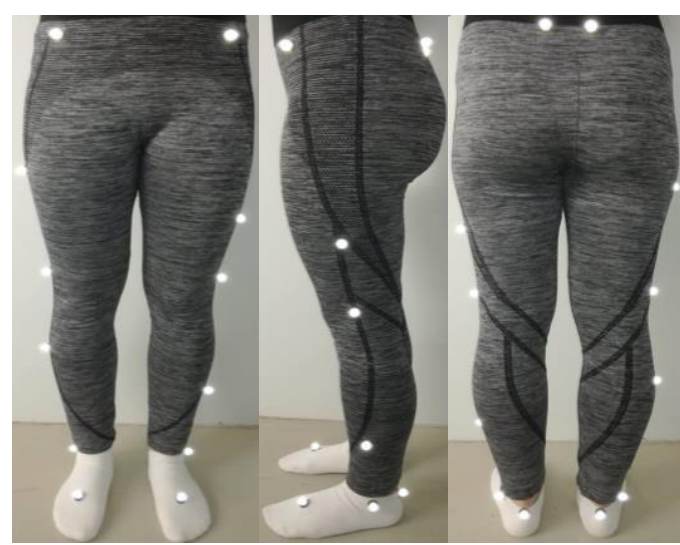

Figure 3. Marker placement adopted for lower limbs.

frequency of $10-\mathrm{Hz}$. Considering that some trials don't have the same number of frames (because some volunteers walk faster than others) it was necessary perform a cubic interpolation to all trials. Thus, all of them have the same number of frames and it is possible analyse the same phase of the gait cycle to all the volunteers.

\section{RESULTS AND DISCUSSION}

Relatively to the data collected with the motion-capture system, it was done a correlation between them and the data from [13]. Fig. 4 represents the mean and the standard deviation (for each trial) of the data collected for two randomly chosen volunteers and the mean of the data of [13]. Visually, there seems to be a good comparation between the data collected and the literature enounced. It is important to note that to the ankle angle of [13] was necessary to add an offset, in order to have an ankle angle according with another references $[\mathbf{1 , 1 4}]$. To have a quantitative value of the correlation existent between the data collected and the literature, it was calculated the Spearman's correlation coefficient which evaluates the strength of a linear monotonic relationship between data. In what concerns to the ankle, knee and hip angles, correlations of $0.90556,0.93578$ and 0.98081 were found, respectively. For the ankle, knee and hip moments, correlations of $0.92341,0.91909$ and 0.89611 were obtained, respectively. Regarding to the GRF, based on the pattern of the Fig. 5, it is possible to identify a good comparation with [15]. It is interesting to analyze the pattern of GRF in comparison with gravitational acceleration, mainly on pre-swing phase (between 50 and $60 \%$ of gait cycle). In this moment, the center of mass is moving downwards, in a normal condition of walking, and this phenomenon implies that an inertial force is added to the gravitational force. Thus, it is expectable that the vertical force on the foot exceeds the gravitational force value $( \pm 5 \%)$. This instant is coincident with the maximum moment exercised on the ankle joint, since it is necessary to boost the body forward [15].

According with the present results, it is plausible to infer that the data collection was done with success because the values obtained for angles and moments are similar to the literature used. However, in some cases, it was noticed that the hip torque presented the lowest values of correlation and analyzing its graph (Fig. 4 - hip moment), some points of inflection are verified. These inflection points cause a decrease in the correlation value because the applied correlation is a linear monotonic correlation. So, the lowest value of 

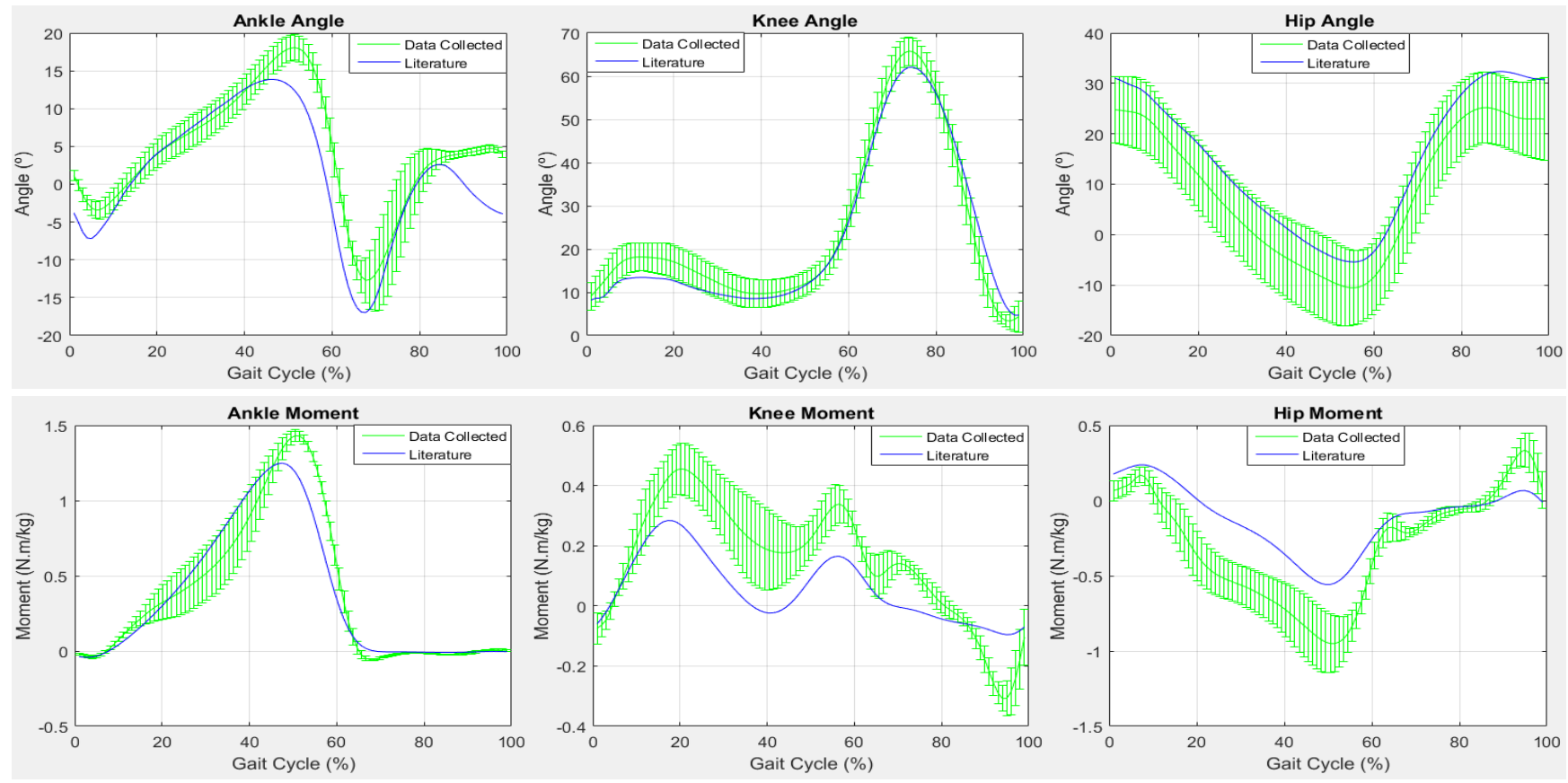

Figure 4. Mean and standard deviation of joint angles and moments for two random chosen volunteers (green) and literature data (blue) available in [13]

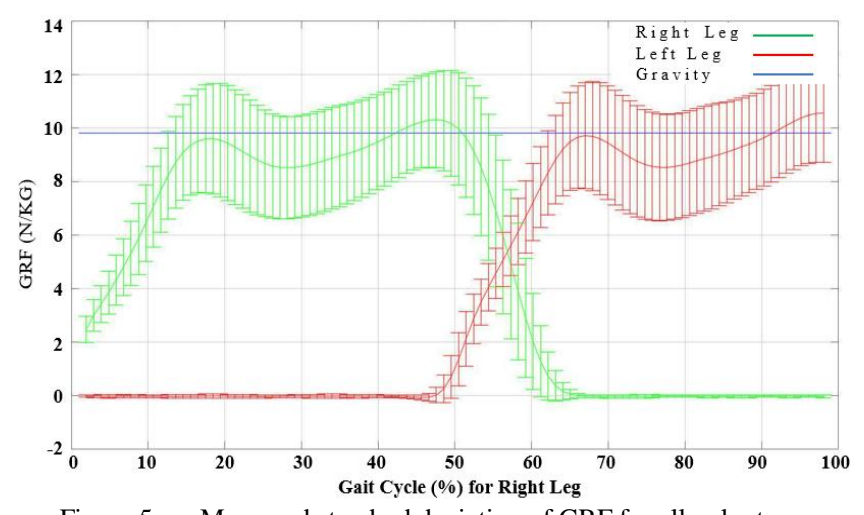

Figure 5. Mean and standard deviation of GRF for all volunteers (green: right leg; red: left leg) normalized by the mean of the weight and gravitational acceleration (blue).

correlation in the hip moment can be related with three aspects: the first is the marker placement, taking in account that the position of the markers is a very delicate step. Another fact that could justify this value is related with the velocity of walking and the pattern of walking. Despite trying to find the best configuration for the force plates, unconsciously, volunteers change their pattern of walking and their speed and this phenomenon can interfere in the calculation of the hip moment. As last alternative to explain this fact, once that was used a lower body configuration, perhaps there is no enough information with respect to the upper body.

\section{CONCLUSION}

In this work are presented the first advances of the construction of a mathematical model to replicate the human gait cycle. The results regarding to the data acquisition are promisors, demonstrating good correlations with the literature. In what concerns to future challenges, these involve the mathematical model validation with the data collected. On the other hand, a study based on the stiffness and power at different walking speeds is also proposed in order to evaluate the energy consumption during this movement.

\section{REFERENCES}

[1] J. Perry and J. M. Burnfield, Gait Analysis: Normal and Pathological Function, Slack Incorporated, 2010, ch. 1, 4, 5 and 6.

[2] A. J. Young and D. P. Ferris, "State of the Art and Future Directions for Lower Limb Robotic Exoskeletons," IEEE Trans. On Neural Systems and Rehabilitation Engineering, vol. 25, no. 2, pp. 171 - 182, Feb. 2017.

[3] A. D. Kuo, "The six determinants of gait and the inverted pendulum analogy: A dynamic walking perspective," Human Movement Science, vol 26, pp. 617 - 656, Jul. 2007.

[4] M. McGrath, D. Howard and R. Baker, "A lagrange-based generalised formulation for the equations of motion of simple walking models," Journal of Biomechanics, vol. 55, pp. 139 - 144, Feb. 2017.

[5] J. H. McDonald, Handbook of Biological Statistics, Sparky House Publishing, 2014, pp. $209-213$.

[6] D. Morin, Introductory Classical Mechanics - with problems and solutions, Cambridge University Press, 2002, ch. 5.

[7] D. A. Winter, Biomechanics and Motor Control of Human Movement, John Wiley \& Sons, 2009, ch. 4. Pp. $82-91$.

[8] Y. P. Lim, Y. Lin,M. G. Pandy, "Effects of step length and step frequency on lower-limb muscle function in human gait," Journal of Biomechanics, vol. 57, pp. 1 - 7, Mar. 2017.

[9] Vicon Motion System, Vicon Nexus User Guide, pp. 99 - 101, Jan 2018.

[10] Vicon Motion System, Plug-in Gait Reference Guide, pp. 6 - 14, Jan. 2018.

[11] A. Pachi and T. Ji, "Frequency and velocity of people walking," The Structural Engineer, pp. 36 - 40, Feb. 2005.

[12] R. Roithner, H. Schwameder and E. Mueller, "Determination of optimal filter parameters for filtering kinematic walking data using butterworth low pass filter," ISBS 2000, Jul. 2000.

[13] G. Bovi, M. Rabuffetti, P. Mazzoleni and M. Ferrarin, "A multipletask gait analysis approach: Kinematic, kinetic and EMG reference data for healthy young and adult subjects," Gait \& Posture, vol. 33, pp. 6 - 13, Aug. 2010.

[14] C. Fukuchi, R. Fukuchi and M. Duarte, "A public dataset of overground and treadmill walking kinematics and kinetics in healthy individuals," PeerJ, Apr. 2018.

[15] T. Marasovic, M. Cecic and V. Zanchi, "Analysis and Interpretation of Ground Reaction Forces in Normal Gait," WSEAS Transactions on Systems, vol. 8, pp. 1105 - 1114, Sep. 2009. 